\title{
Oil-based cyclo-oligosaccharide nanodevices for drug encapsulation
}

\author{
Jennifer Noro $^{\mathrm{a}}$, Ana Loureiro ${ }^{\mathrm{a}}$, Filipa Gonçalves ${ }^{\mathrm{a}}$, Nuno G. Azoia ${ }^{\mathrm{a}}$, Seunho Jung ${ }^{\mathrm{b}}$, \\ Carla Silva ${ }^{a}$, Artur Cavaco-Paulo ${ }^{\mathrm{a}, *}$ \\ a Centre of Biological Engineering, University of Minho, Campus de Gualtar, 4710-057, Braga, Portugal \\ b Department of Microbial Engineering and Bio/Molecular Informatics Center, Konkuk University, 1 Hwayang-dong, Gwangjin-gu, Seoul 143-701 South \\ Korea
}

\section{A R T I C L E I N F O}

\section{Article history:}

Received 22 May 2017

Received in revised form 14 July 2017

Accepted 24 July 2017

Available online 25 July 2017

\section{Keywords:}

Cyclo-oligosaccharides

Cyclosophoraose

Palmitoyl chloride

Phase-change

Nanoemulsions

Ultrasound

\begin{abstract}
A B S T R A C T
New encapsulation nanodevices were synthesized by emulsification of cyclo-oligosaccharides fully substituted by hydrophobic palmitic chains. These highly hydrophobic compounds, acquire oily-like behaviour at moderate temperatures $\left(\sim 50^{\circ} \mathrm{C}\right)$ and when submitted to ultrasound (US) can undergo emulsification. The improved emulsifying properties of modified cyclo-oligosaccharides are suitable to produce small and narrow sized nanoemulsions with ability to encapsulate amphiphilic molecules. Both encapsulation and delivery of a therapeutic drug, methotrexate (MTX), with amphiphilic character was assessed. The physicochemical properties of the cyclo-oligosaccharide nanoemulsions containing MTX were investigated by nuclear magnetic resonance (NMR), scanning transmission electron microscopy (STEM) and dynamic light scattering (DLS). The results revealed that the modified cyclo-oligosaccharides are potential platforms for the encapsulation of bio compounds for cosmetic and pharmaceutical purposes.
\end{abstract}

(C) 2017 Elsevier B.V. All rights reserved.

\section{Introduction}

The administration of drugs with poor solubility and low rate of dissolution through different routes has been a major challenge for the pharmaceutical research. Several techniques have been attempt to drive drugs solubility, bioavailability and dissolution properties, namely solubilisation [1,2], cosolvency [3] and solid dispersions $[4,5]$. The encapsulation of drugs into nanodevices has also been explored to overcome the unfavourable solubility and inappropriate interactions of drugs with other chemical compounds during delivery that are responsible by their toxicological characteristics [6]. Moreover, the nanodevices may protect the drug from degradation and increase its selectivity by modifying the pharmacokinetic and drug tissue distribution profile, which ensure a high safety and biocompatibility [6,7]. Among various particulate systems, liposomes and nanoparticles have been applied for site specific delivery of drugs. However, they possess size dependent properties, stability and scale-up problems. Lipid-based delivery systems and water-in-oil microemulsions have overcome the frequency of administration of certain drugs, e.g. MTX, by providing the control delivery to absorption sites but any of them was able to achieve

\footnotetext{
* Corresponding author.

E-mail address: artur@deb.uminho.pt (A. Cavaco-Paulo).
}

the target or minimize the adverse side effects [8]. The encapsulation of drugs has been explored by using several other devices, namely micellar nano-networks[9], magnetic microcapsules [10], PLA-PEG-PLA nanoparticles [11], dendrimeric nanodevices [12], protein nanoemulsions $[13,14]$ among others. Still, some of them showed an almost completely release of the drug after one day $[9,10]$. Cyclo-oligosaccharides complexation has been presented as a promising strategy to tackle the mentioned formulation issues. Their ability to form inclusion complexes with a wide variety of hydrophobic compounds provide significant advantages related with changes of the physicochemical and biological properties of the guest molecules. The complexation can also provide protection of the component against light and oxidation, its solubilisation, handling improvement and stability [15]. Cyclosophoraoses (Cys) are unbranched cyclic $\beta$-1,2-D-glucans containing 17-23 glucose residues produced by Rhizobium and Agrobacterium species both intracellularly and extracellularly [16]. The targeting of non-polar chemicals like ergosterol, luteolin, vitamin D3 and naproxen has been reported as Cys complexation examples [17,18]. A flexible backbone structure with doughnut-like ring shape is attributed to Cys and is responsible for the atypical induced-fit type complexation with hydrophobic molecules [16]. Besides complexation systems, Cys can also be applied as a novel biosourced saccharide catalyst for chemical reactions as reported by Dindulkar et al. [19]. They reported the use of microbial Cys for the synthesis of ther- 
apeutically important versatile indolyl $4 \mathrm{H}$-chromenes via a one pot three-component Knoevenagel-Michael addition-cyclization reaction of salicylaldehyde, 1,3-cyclohexanedione/dimedone, and indoles in water under neutral conditions.

The chemical modification of Cys has been also assessed by Kim et al., to increase its hydrophobicity [20]. Their studies revealed the improvement of the solubility of insoluble flavonoids, comparing with the non-modified Cys [20]. They also described a new pentacosadiynoyl cyclosophoraose (P-Cys) synthesized using a biosourced cyclo-oligosaccharide with intrinsic complexation capacity [16]. Their results indicated that P-Cys is a useful platform for encapsulating emulsification of bioactive molecules for cosmetic and pharmaceutical applications.

The main aims of this study were firstly to chemically modify the cyclo-oligosaccharides (Cys, $\beta$-cyclodextrin (CD) and $\gamma$-CD) by reaction of the hydroxyl groups with an hydrophobic compound, palmitoyl chloride (PAL-Cl), and to produce cyclo-oligosaccharidePAL-based nanoemulsions via ultrasound-assisted methodology. This methodology has been previously exploited by Silva et al. [21] and Loureiro et al. [13]. Microspheres of bovine serum albumin (BSA) and silk fibroin were produced by applying ultrasound in a biphasic system consisting of an aqueous protein solution and an organic solvent. The protein microspheres are dispersed in an aqueous media where the protein remains at the interface covering the organic solvent. These nanodevices can improve the solubility of hydrophobic drugs encapsulated in the organic phase of the particles. The modified Cys hold typical fusion properties (melting point of around $50^{\circ} \mathrm{C}$ ) which in turns powder into a viscous liquid. Taking advantage of this phase-change, nanoemulsions of Cys-PAL and Cys-PAL/BSA were produced applying ultrasound in a biphasic system consisting of an aqueous solution (BSA/buffer) and an organic phase (Cys-PAL). The overall physicochemical and biological characterization of the developed nanoemulsions were performed. A comparison with other cyclo-oligosaccharides, namely $\beta$ - and $\gamma$ CD was assessed. MTX was used as a model guest molecule to test the entrapment ability of the developed nanodevices. The efficiency of entrapment and the drug release over time were evaluated by fluorescence microscopy.

\section{Materials and methods}

All compounds were purchase from Sigma-Aldrich or TCI chemicals, and used without further purifications. Column chromatography was made using silica gel $60 \mathrm{~A}$, with particle size of $70-200 \mu \mathrm{m}$ as stationary phase, and ethyl acetate, chloroform or ethanol as mobile phase for the separation of the components. TLC plates (silica gel $60 \mathrm{~F}_{254}$ ) were revealed under a UV lamp. Human skin fibroblasts (BJ5ta cell line) (ATCC, CRL-4001) were obtained from American Type Culture Collection (LGC Standards, UK) and all culture media and supplements were purchased from SigmaAldrich. T75 flasks and 96-well tissue culture polystyrene plates were purchase from TPP, Switzerland. MTS assay was acquired from Promega, USA. MTX sodium salt was purchased from TevaGuard pharmaceutical.

\subsection{Isolation of cyclosophoraose}

Cys (cyclic $\beta-1,2$ glucans) was isolated from Rhizobium leguminosarum biovar viciae VF39 following the procedures found in the literature [22,23]. MALDI-TOF and NMR techniques were applied to confirm the structure and the average molecular weight.

\subsection{Synthesis and characterization of modified cyclo-oligosaccharides}

The modified cyclo-oligosaccharides were all synthetized using a similar procedure. Briefly, to a solution of cyclo-oligosaccharide (84-125 mg, 0.027-0.106 mmol) in dry pyridine $(4-5 \mathrm{~mL})$ under nitrogen atmosphere, was dropped the PAL-Cl $(0.5-1.0 \mathrm{~mL}, 1.65-$ $3.30 \mathrm{mmol}$ ) using a water bath at $50^{\circ} \mathrm{C}$. The mixture was stirred overnight at the same temperature. The solvent was removed by co-evaporation with toluene in a rotary evaporator. The formed solid was further purified by column chromatography to obtain the pure product $(0.35-0.48 \mathrm{~g}, \mathrm{y}=60.4-74.9 \%)$.

Cys ( $84 \mathrm{mg}, 0.027 \mathrm{mmol}) ;$ PAL-Cl $(0.5 \mathrm{~mL}, 1.65 \mathrm{mmol})$; Dry pyridine $(5 \mathrm{~mL}) ; 50^{\circ} \mathrm{C}$; overnight. Column chromatography $50 \%$ $\mathrm{CHCl}_{3} / 50 \%$ AcOEt. Beige solid $(0.35 \mathrm{~g}, 0.020 \mathrm{mmol}, 74.9 \%)$. Melting point $=48-50{ }^{\circ} \mathrm{C} . \delta_{\mathrm{H}}\left(\mathrm{CDCl}_{3}\right): 0.88(\mathrm{t}, J=6.8 \mathrm{~Hz}), 1.28(\mathrm{br} \mathrm{s}), 1.63(\mathrm{~m})$, 2.35 (t, $J=7.6 \mathrm{~Hz}$ ), 3.0-5.5 (br s) ppm. MALDI-TOF $20045 \mathrm{~m} / \mathrm{z}$.

及-CD $(120 \mathrm{mg}, 0.106 \mathrm{mmol}) ; \mathrm{PAL}-\mathrm{Cl}(1 \mathrm{~mL}, 3.30 \mathrm{mmol})$; Dry pyridine $(4 \mathrm{~mL}) ; 50{ }^{\circ} \mathrm{C}$; overnight. Column chromatography $\mathrm{CHCl}_{3} / \mathrm{EtOH}$ (6:1). Beige solid (0.392 g, $\left.0.064 \mathrm{mmol}, 60.4 \%\right)$. Melting point $=44-46^{\circ} \mathrm{C} . \delta_{\mathrm{H}}\left(\mathrm{CDCl}_{3}\right): 0.88(\mathrm{t}, J=6.8 \mathrm{~Hz}), 1.25(\mathrm{br} \mathrm{s}), 1.62$ (m), $2.34(\mathrm{~m}), 3.49(\mathrm{~m}), 3.97(\mathrm{~m}), 4.10(\mathrm{~m}), 4.51(\mathrm{~m}), 4.95(\mathrm{~m}) \mathrm{ppm}$.

$\boldsymbol{\gamma}$-CD (125 mg, $0.096 \mathrm{mmol}) ;$ PAL-Cl $(0.9 \mathrm{~mL}, 2.31 \mathrm{mmol})$; Dry pyridine $(5 \mathrm{~mL}) ; 50{ }^{\circ} \mathrm{C}$; overnight. Column chromatography $50 \%$ $\mathrm{CHCl}_{3} / 50 \%$ AcOEt. Beige solid $(0.48 \mathrm{~g}, 0.068 \mathrm{mmol}, 70.7 \%)$. Melting point $=49-50{ }^{\circ} \mathrm{C} . \delta_{\mathrm{H}}\left(\mathrm{CDCl}_{3}\right): 0.89(\mathrm{t}, J=6.8 \mathrm{~Hz}), 1.31(\mathrm{br} \mathrm{s}), 1.63(\mathrm{~m})$, $2.36(\mathrm{~m}), 3.50(\mathrm{~m}), 3.87(\mathrm{~m}), 4.01(\mathrm{~m}), 4.14(\mathrm{~m}), 4.48(\mathrm{~m}), 4.90(\mathrm{~m})$, $5.03(\mathrm{~m}) \mathrm{ppm}$.

All the modified cyclo-oligosaccharides were characterized by ${ }^{1} \mathrm{H}$ NMR spectroscopy, using a Bruker Avance III $400(400 \mathrm{MHz})$. $\mathrm{CDCl}_{3}$, DMSO- $d_{6}$ or $\mathrm{D}_{2} \mathrm{O}$ were used as deuterated solvents, using the peak solvent as internal reference. Multiplicities are indicated as: $t$ as triplet, $b r s$ as broad singlet and $m$ as multiplet. Melting points were determined using a Gallenkamp apparatus. MALDI-TOF mass spectra were acquired on a Bruker Autoflex Speed instrument (Bruker Daltonics GmbH) equipped with a $337 \mathrm{~nm}$ nitrogen laser. The matrix solution for MALDI-MS measurement was prepared by dissolving a saturated solution of 2,5-dihydroxybenzoinc acid (DHB) in $100 \%$ ethanol. Samples were spotted onto a ground steel target plate (Bruker part $n^{\circ}$ 209519) and analysed in the linear positive mode using factory-configured instrument parameters suitable for a 10-30 kDa $m / z$ range (ion source $1: 19,5 \mathrm{kV}$; ion source $2: 18,3 \mathrm{kV}$ ). Time delay between laser pulse and ion extraction was set to $130 \mathrm{~ns}$, and the laser frequency was $25 \mathrm{~Hz}$.

\subsection{Cyclo-oligosaccharide-palmitoyl conjugate nanoemulsions}

The nanoemulsions composed of cyclo-oligosaccharide (alone or in the presence of BSA) were produced by application of US. The mixtures containing the cyclo-oligosaccharide (alone or in presence of BSA) ( $4 \mathrm{~mL}$ ) in phosphate buffer saline (PBS) $10 \mathrm{mM}$ at $\mathrm{pH}=7.4$ were previously submitted to a pre-heat at $60^{\circ} \mathrm{C}$ for $10 \mathrm{~min}$. Then, the mixture was subjected to US using a high-intensity ultrasonic horn $(20 \mathrm{KHz}$ Sonics \& Materials Vibracell CV 33, $3 \mathrm{~mm}$ diameter titanium microtip) positioned at the middle of the mixture. The ultrasonication was performed at $60^{\circ} \mathrm{C}$ for $3 \mathrm{~min}$ with an amplitude of $40 \%$. Control samples with unmodified oligosaccharides were also performed using the same conditions.

\subsection{Dynamic light scattering}

The mean diameter ( $\mathrm{nm}$ ), polydispersity index (PDI) and zetapotential $(\mathrm{mV})$ of the nanoemulsions were measured in a Zetasizer Nano ZS (Malvern Instruments) at $25^{\circ} \mathrm{C}$. Prior to DLS measurements, the samples were diluted with PBS at pH 7.4 (for size) and with ultrapure water (for zeta-potential). In both cases, a 
dilution of $5 \mathrm{x}$ was made, followed by a filtration using $0.45 \mu \mathrm{m}$ filters, (Spartan ${ }^{\mathrm{TM}} 30 \mathrm{RC}$, Whatman, GE Healthcare). All measurements were read in triplicate, being the results described as mean \pm standard deviation.

\subsection{Nanoparticle tracking analysis (NTA)}

NTA was used to access quantitatively the obtained nanoemulsions. The experiments were performed using a NanoSight NS500 instrument (Salisbury, UK). This system includes a charge coupled device (CCD) camera that allows visualization and tracking Brownian motion of laser-illuminated particles in suspension. The measurements were made at room temperature and each video sequence was captured over $60 \mathrm{~s}$. The samples were $10 \times$ diluted with water and filtered (Millipore filters with pore size of $0.45 \mu \mathrm{m}$ ) and then injected into the system.

\subsection{Differential scanning calorimetry (DSC)}

All measurements were conducted on a power-compensated DSC instrument (DSC 6000 Perkin Elmer) with a nitrogen flux of $20 \mathrm{~mL} / \mathrm{min}$, using stainless steel capsules in the temperature range of $20-100^{\circ} \mathrm{C}$ (heating rate: $10^{\circ} \mathrm{C} / \mathrm{min}$, powder sample weight: 3-7 mg). The DSC device was calibrated using indium and zinc, both of high purity. The samples were stored at selected levels of humidity (relative humidity, $\mathrm{RH}$, of $45 \%$ ) and temperature $\left(20-22{ }^{\circ} \mathrm{C}\right.$ ) for $24 \mathrm{~h}$ prior to the analyses and each sample was measured at least two times, in order to validate the results.

\subsection{Scanning transmission electron microscopy (STEM)}

STEM was performed with a NOVA Nano SEM 200 FEI microscope. The carbon coated copper grids (400 meshes, $3 \mathrm{~mm}$ diameter) were placed in contact with the nanoemulsions and the excess of solvent was instantly absorbed by a filter paper. Another set of samples was prepared by the same way with the addition of a final staining with uranyl acetate. For this, the grids were placed on the top of a drop of uranyl acetate $(0.2 \% \mathrm{v} / \mathrm{w})$ for $5 \mathrm{~min}$. The excess of the liquid was removed as described above and the grids were washed with water and dried at room temperature.

\subsection{Molecular dynamics simulations}

The simulations were performed with the software package GROMACS, using Martini force-field [24]. The system size was chosen according to the minimum image convention taking into account a cut-off of $1.2 \mathrm{~nm}$. The bonds lengths were con-strained with LINCS [25]. Non-bonded interactions were calculated using a twin-range method, with short and long range cut-offs of 0.9-1.2 nm, respectively. Neighbour searching was carried out up to1.2 $\mathrm{nm}$ and updated every ten steps. A time step of integration of $5 \mathrm{fs}$ was used. A reaction field correction for the electrostatic interactions was applied using a dielectric constant of 15. Pressure control was implemented using the Berendsen barostat [26], with a reference pressure of $1 \mathrm{bar}, 3.0 \mathrm{ps}$ of relaxation time and isothermal compressibility of $3.0 \times 10^{-5}$ bar $^{-1}$. Temperature control was set using the Berendsen thermostat [26] at $520 \mathrm{~K}$. Each component of the system was included in separated heat bath, with temperatures coupling constants of $0.30 \mathrm{ps}$. Two replica simulations of $15 \mathrm{~ns}$ in length were carried out using different initial velocities taken from a Maxwell-Boltzman distribution at $420 \mathrm{~K}$. Initially the components were distributed uniformly through the simulation box and allowed to interact freely during the simulation.

\subsection{Cellular viability assay}

\subsubsection{Cells and culture conditions}

BJ5ta cell line, an adherent cell line, was grown in T75 flasks using a medium composed by 4 parts of Dulbeccoís Modified Eagle's Medium (DMEM) and 1 part of M199 Medium supplemented with 2 mM L-glutamine; $4.5 \mathrm{~g} / \mathrm{L}$ glucose; $1.5 \mathrm{~g} /$ L sodium bicarbonate; $10 \%$ $(\mathrm{v} / \mathrm{v})$ of fetal bovine serum (FBS); $1 \%(\mathrm{v} / \mathrm{v})$ of penicillin/streptomycin solution; $10 \mu \mathrm{g} / \mathrm{mL}$ of hygromycin B. Exponentially growing culture was maintained in a humidified atmosphere of $5 \% \mathrm{CO}_{2}$ in air at $37^{\circ} \mathrm{C}$.

\subsubsection{Cell viability assay}

Cell viability was studied using the Promega CellTiter $96{ }^{\circledR}$ AQueous Non-Radioactive Cell Proliferation (MTS) assay. BJ5ta cells were seeded in 96-well tissue culture polystyrene plates at a density of $1 \times 10^{4}$ cells/well and incubated overnight to promote cell adhesion. The cells were incubated with different concentrations of nanoparticles (from $0.0625 \mathrm{mg} / \mathrm{mL}$ up to $1 \mathrm{mg} / \mathrm{mL}$ ). The concentrations of nanoemulsions for the application in cells were calculated based in the concentration of cyclo-oligosaccharides in the initial formulations $(5 \mathrm{mg} / \mathrm{mL})$. The nanoemulsions were incubated with cells for 24,48 and $72 \mathrm{~h}$. A MTS mixture was then added and the cells were further incubated for $4 \mathrm{~h}$ at $37^{\circ} \mathrm{C}$. After this period, the plates were placed on Synergy Mx Multi-Mode Reader from BioTek (USA) and the absorbance of the formazan product was read at $490 \mathrm{~nm}$. Cell viability was expressed as a percentage relative to the negative control (untreated control cells). Two independent experiments were made.

\subsection{Stability of nanoemulsions in the presence of lipase}

The disruption of the produced nanoemulsions was assessed by incubation with a esterase, namely lipase. For this, $1 \mathrm{~mL}$ of each sample was transferred to an assay tube, and incubated with lipase from Thermomyces Lanuginosus (activity: $407 \mu \mathrm{mol} / \mathrm{mL} / \mathrm{min}$ ) $(50 \mu \mathrm{L})$ at $40^{\circ} \mathrm{C}$ in a water bath at $60 \mathrm{rpm}$ for $48 \mathrm{~h}$. Aliquots were taken at different time points to measure the size of nanoemulsions and confirm disruption. Prior to DLS measurement the samples were diluted (5x) and filtered with $0.45 \mu \mathrm{m}$ filter. The final disruption of nanoemulsions was evaluated after $48 \mathrm{~h}$ of incubation by extraction of the samples with $\mathrm{CHCl}_{3}(3 \times 10 \mathrm{~mL})$. The organic layer was dried over magnesium sulphate and filtered. The solution was evaporated using a rotary evaporator. The NMR spectra was acquired firstly in DMSO- $d_{6}$, to detect the presence of palmitic acid. Afterwards, the NMR was acquired in $\mathrm{CDCl}_{3}$ to evaluate the percentage of nanoemulsions ester cleavage.

\subsection{Encapsulation and release of methotrexate}

The ability of the developed nanoemulsions to encapsulate MTX was evaluated. The encapsulation was performed by including $10 \mathrm{mg} / \mathrm{mL}$ of MTX in the aqueous phase of nanoemulsions composition. The final nanodevices were produced as previously described in Section 2.3. The encapsulation efficiency was verified by NMR spectroscopy by evaluation of the interaction between MTX and the modified cyclo-oligosaccharides. This evaluation was assessed by the chemical shit analysis comparing to the MTX in the free form. The MTX release profile was evaluated by placing the nanoemulsions containing MTX in mini dialysis tubes with $1 \mathrm{kDa}$ cut-off (GE Healthcare) against PBS ( $1 \mathrm{mM})$. Aliquots were taken at different time points and read in a 96-quartzo microplate at $303 \mathrm{~nm}$ for $72 \mathrm{~h}$. After this period the PBS was replaced and the release proceed until no more MTX was detected (192 h). 
A

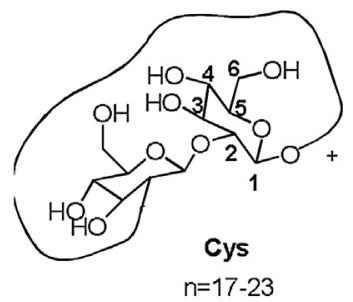

$n=17-23$

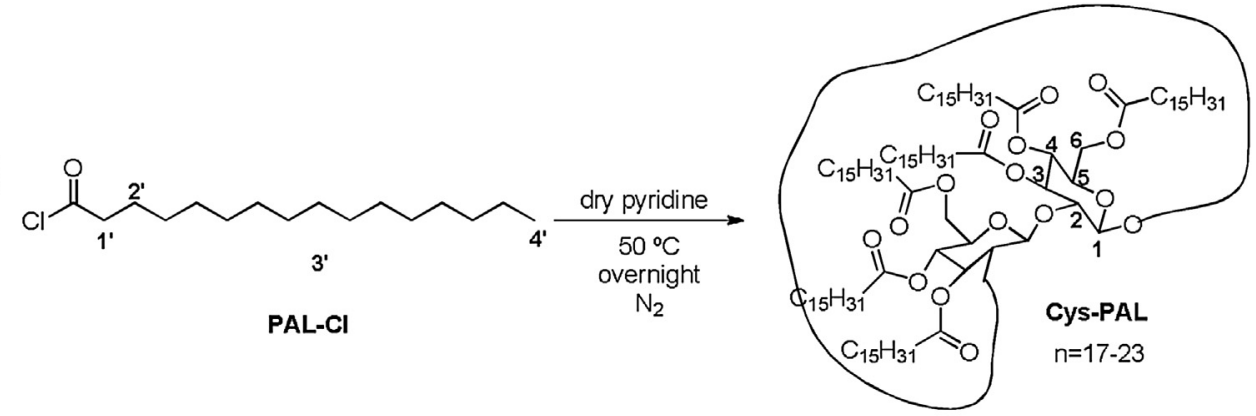

B

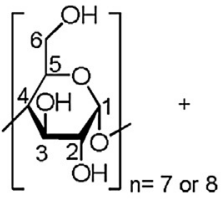

CD

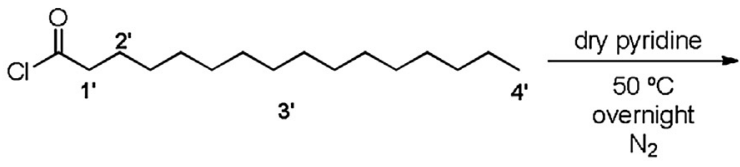

PAL-CI

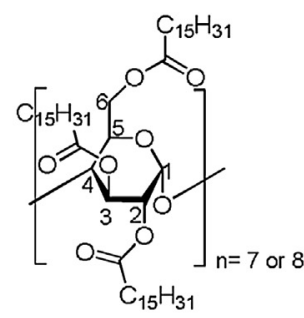

CD-PAL

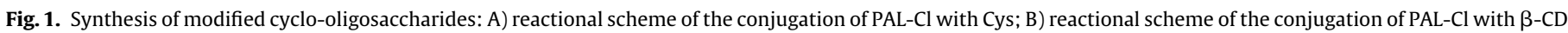
$(\mathrm{n}=7)$ and $\gamma-\mathrm{CD}(\mathrm{n}=8)$.

\section{Results and discussion}

\subsection{Synthesis of hydrophobic cyclo-oligosaccharides by conjugation with palmitoyl chloride}

The synthesis of cyclo-oligosaccharides-PAL conjugates was carried out as previously described (Fig. 1). A fully substitution of the hydroxyl groups of cyclo-oligosaccharides by PAL was accomplished by performing the reaction at $50^{\circ} \mathrm{C}$, overnight in the presence of pyridine. The isolation and purification of the final products was assessed using a chromatography column to separate the pyridinium chloride sub-product. High yields of product synthesis were obtained ( $y=60.4-74.9 \%$ ).

The molecular weight of modified cyclo-oligosaccharides was determined by MALDI-TOF mass spectrometry (see supplementary data, Fig. S.1). Based on the degree of polymerization (DP) of the cyclo-oligosaccharides, the ionization patterns of synthesized cyclo-oligosaccharides-PAL were determined as described in Table S.1. Cys has a number average molecular weight (Mn) of 3134, Cys-PAL shows the substituted mass obtained according to the corresponding DP (23) conjugated with 68 PAL units. The molecular ions of $\beta$-CD-PAL are represented as [DP7 + $11-17$ PAL], with signals at $m / z 3799,4016,4253,4493,4733,4963$ and 5200 . The representative ions of $\gamma$-CD-PAL are represented as [DP8+13-20PAL], with signals at $m / z 4422,4660,4900,5138$, $5378,5614,5853$ and 6090 (Table S.1). The mass difference between cyclo-oligosaccharides and cyclo-oligosaccharides-PAL is $\mathrm{m} / \mathrm{z} 240$ attributed to the palmitoyl chain.

The structures of the modified cyclo-oligosaccharides were elucidated recording the ${ }^{1} \mathrm{H} \mathrm{NMR}$ in $\mathrm{CDCl}_{3}$ (Fig. 2).

The presence of the palmitic chain is confirmed by the peaks assigned as $1^{\prime}$ to $4^{\prime}$. Peak $4^{\prime}$ is the terminal $\mathrm{CH}_{3}$ of the palmitic chain, arising as a triplet near $\delta_{\mathrm{H}} 0.9 \mathrm{ppm}$ in all cases. $1^{\prime}$ and $2^{\prime}$ are the $\mathrm{CH}_{2}$ next the carbonyl group respectively, being $1^{\prime}$ a triplet or multiplet $\left(\delta_{\mathrm{H}} 2.34,2.35\right.$ or $2.36 \mathrm{ppm}$ ), and $2^{\prime}$ a multiplet ( $\delta_{\mathrm{H}} 1.62$ or $1.63 \mathrm{ppm})$. The chain of palmitoyl appears as a multiplet numbered as $3^{\prime}\left(\delta_{\mathrm{H}} 1.25,1.28\right.$ or $\left.1.31 \mathrm{ppm}\right)$. In the $\beta-C D$ and $\gamma-C D$ is possible to calculate the substitution degree regarding the relation between one of the peaks of the oligosaccharides and the $\mathrm{CH}_{3}$ of the palmitoyl. This confirmed the full substitution of both compounds by palmitic chains. For Cys-PAL it is possible to observe that all $\mathrm{C}-\mathrm{H}$ of the oligosaccharide scaffold appeared as a broad singlet between $\delta_{\mathrm{H}} 3.0$ and $5.5 \mathrm{ppm}$, hindering the calculation of the substitution degree. Considering $\beta$ - and $\gamma-C D$, the $C-H$ peaks of the oligosaccharide units are observed as multiplet signals in both cases $(\beta-C D$ between $\delta_{\mathrm{H}} 3.49-4.95 \mathrm{ppm} ; \gamma$-CD between $\left.\delta_{\mathrm{H}} 3.50-5.03 \mathrm{ppm}\right)$.

\subsection{Cyclo-oligosaccharides-palmitoyl emulsification ability}

The formation of narrow sized and stable nanoemulsions containing $\mathrm{BSA} / \mathrm{n}$-dodecane by ultrasonication was previously investigated by Silva et al. [21]. They postulate that in an aqueous phase, proteins form stable 3D structures based on the balance between the outer hydrophilic segments covering the inner hydrophobic segments in a conformation of minimal energy. When high shear forces like US are applied to the biphasic system of water/oil, the proteins tend to adapt their structure and migrate to the interface. Depending on the protein structure, its adaptation may include a 3D modification of the protein [21]. In all cases, the protein seems to form a shell with hydrophobic characteristics near the organic solvent and hydrophilic characteristics near water. The protein microspheres are dispersed in the aqueous media whereas the protein remains at the interface covering the organic solvent [21]. Herein, we present a similar approach for the production of nanoemulsions of cyclo-oligosaccharides however excluding the organic solvent from the system. Experiments containing different concentrations of BSA on nanoemulsions composition were also performed since this protein is a well-known material for nanoparticles production and drug encapsulation.

The emulsification ability of modified cyclo-oligosaccharides was investigated through the formation of emulsions in cyclooligosaccharide/water mixture system. The modified cyclooligosaccharides are in a powder form and when heated at $60^{\circ} \mathrm{C}$ (m.p. $=43-60^{\circ} \mathrm{C}$ ) (see Fig. S.2) they acquire oily-like behaviour rendering to the system self-assembly capacity. We found that the oilin-water nanoemulsions formed by cyclo-oligosaccharide/water 
A Cys-PAL

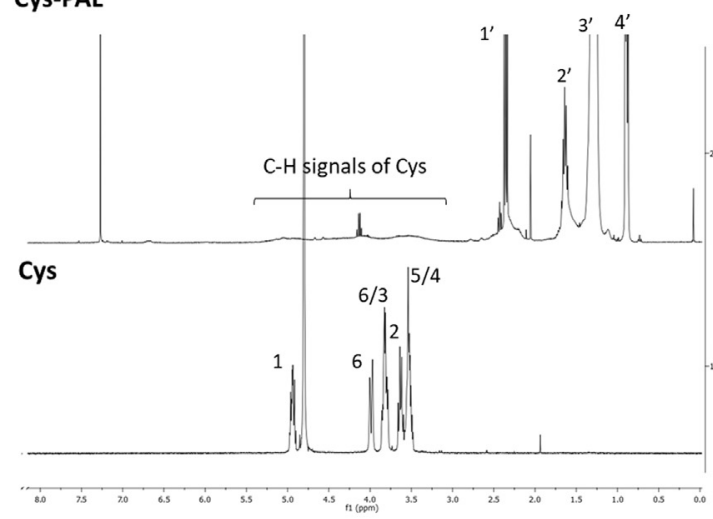

C

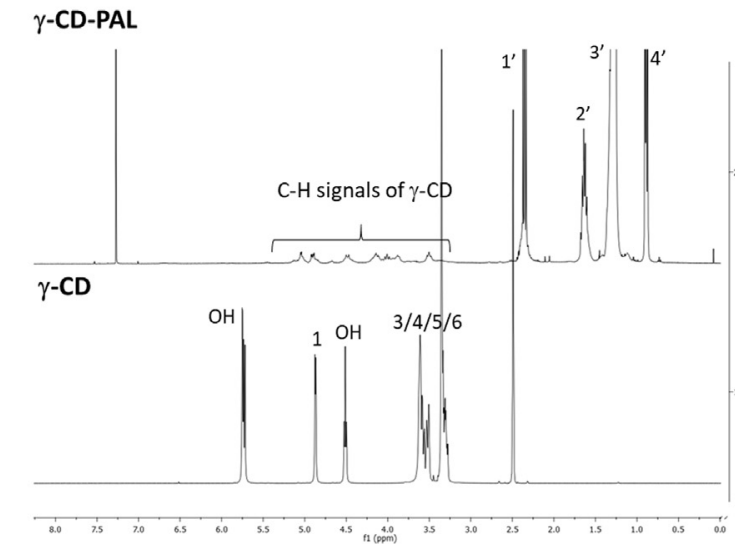

B B-CD-PAL

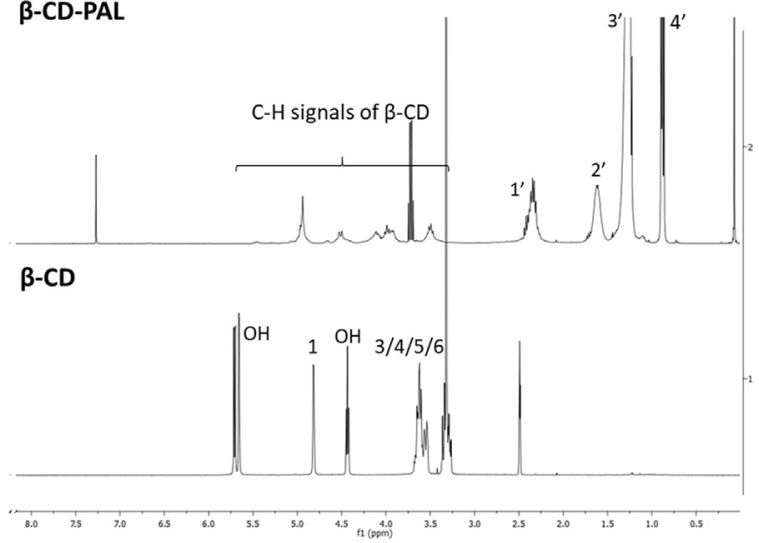

Fig. 2. NMR spectra of (A) Cys and Cys-PAL; (B) $\beta-C D$ and $\beta-C D-P A L$ and $(C) \gamma-C D$ and $\gamma$-CD-PAL.

exhibit remarkable stability against coalescence with the exception of $\beta$-CD-PAL. All of these observations suggest an increased selfassembly ability of cyclo-oligosaccharide containing more than 7 sugar units, which is incremented by the conjugation with the hydrophobic palmitoyl units. The self-assembly properties can be attributed to the characteristic flexible conformation of cyclooligosaccharides applied. It is noteworthy to highlight that with unmodified oligosaccharides no formation of nanoemulsions was observed (Fig. S.3a). Their entrapment ability can be combined with the interaction of the conjugated hydrophobic tail, where the emulsification and encapsulation are differentiated depending on the host structures. The developed devices are good candidates for the encapsulation of amphiphilic substances.

Molecular dynamics simulations, used herein to better understand the interactions between all the components of the nanoemulsions, corroborate our assumptions confirming the ability of Cys-PAL and BSA-Cys-PAL to undergo emulsification. From the data obtained it is evident the interaction between the hydrophobic part of BSA and the oily-like phase of Cys-PAL, as it can be depicted in Fig. 3B (III and IV). These results also allow us to speculate that this behaviour would decrease hydrophobic interactions between Cys-PAL and BSA, maybe contributing for the size reduction when BSA is present in the nanoemulsions (Fig. 3A). The auto aggregation properties of Cys-PAL can justify the bigger sizes observed by DLS (Fig. 3A).

\subsubsection{Microscopic observation}

The morphology and structure of the developed nanoemulsions (Cys-PAL, $\beta$-CD-PAL, $\gamma$-CD-PAL, BSA-Cys-PAL, BSA- $\beta-C D-P A L$ and BSA- $\gamma$-CD-PAL) were investigated by STEM to assess the form and the size of emulsion droplets (Fig. S.3). All the nanoemulsions present homogeneous and regular aspect with particle size between 100 and $200 \mathrm{~nm}$, with the exception of $\beta$-CD-PAL and BSA$\beta$-CD-PAL (data not shown). These samples present much lower particle size compared with the others and by eye visualization a low stability against flocculation and coalescence is observed. The amount of sugar units played here a limiting role by hindering the nanoemulsions formation when $\beta-C D$ ( 7 sugar units) was used. For further experiments, we exclude these samples from the studies. The results also demonstrate that Cys-PAL, $\gamma$-CD-PAL, BSA-Cys-PAL and BSA- $\gamma$-CD-PAL were successfully converted into spherical and smooth nanoemulsions by using high intensity ultrasound. This morphology is expected to offer high encapsulation potential as well as high release performance and drug protection, providing the minimum contact with the aqueous environment [21].

\subsubsection{Nanoemulsions stability over time}

The relative homogeneity of the developed nanoemulsions was further investigated by DLS analysis. Fig. $3 \mathrm{~A}$ presents the mean size and PDI of samples at the first day of production and after 60 days of storage at $4{ }^{\circ} \mathrm{C}$. All the formulations present narrow sized distribution between 100 and $200 \mathrm{~nm}$ in diameter with low PDI (0.1-0.3) remaining stable in nanoemulsions state at least for 60 days of storage at $4{ }^{\circ} \mathrm{C}$. Even though the values of size slightly changed a long time, the PDI values remain highly stable, which is a good indicator for future applications. The zeta-potential is an important parameter in nanoemulsions characterization which allows to predict their stability over time. Long term stability of produced nanoemulsions has been verified, which is in accordance with the zeta-potential data obtained. The nanoparticles are highly negatively charged indicating high repulsive forces with low probability of agglomeration. Cys-PAL and $\gamma$-CD-PAL present similar zeta-potential values $(\approx-40 \mathrm{mV}$ ) while BSA-Cys-PAL and BSA- $\gamma$-PAL show zeta-values of around $-20 \mathrm{mV}$ attributed to the complexation of the cyclo- 


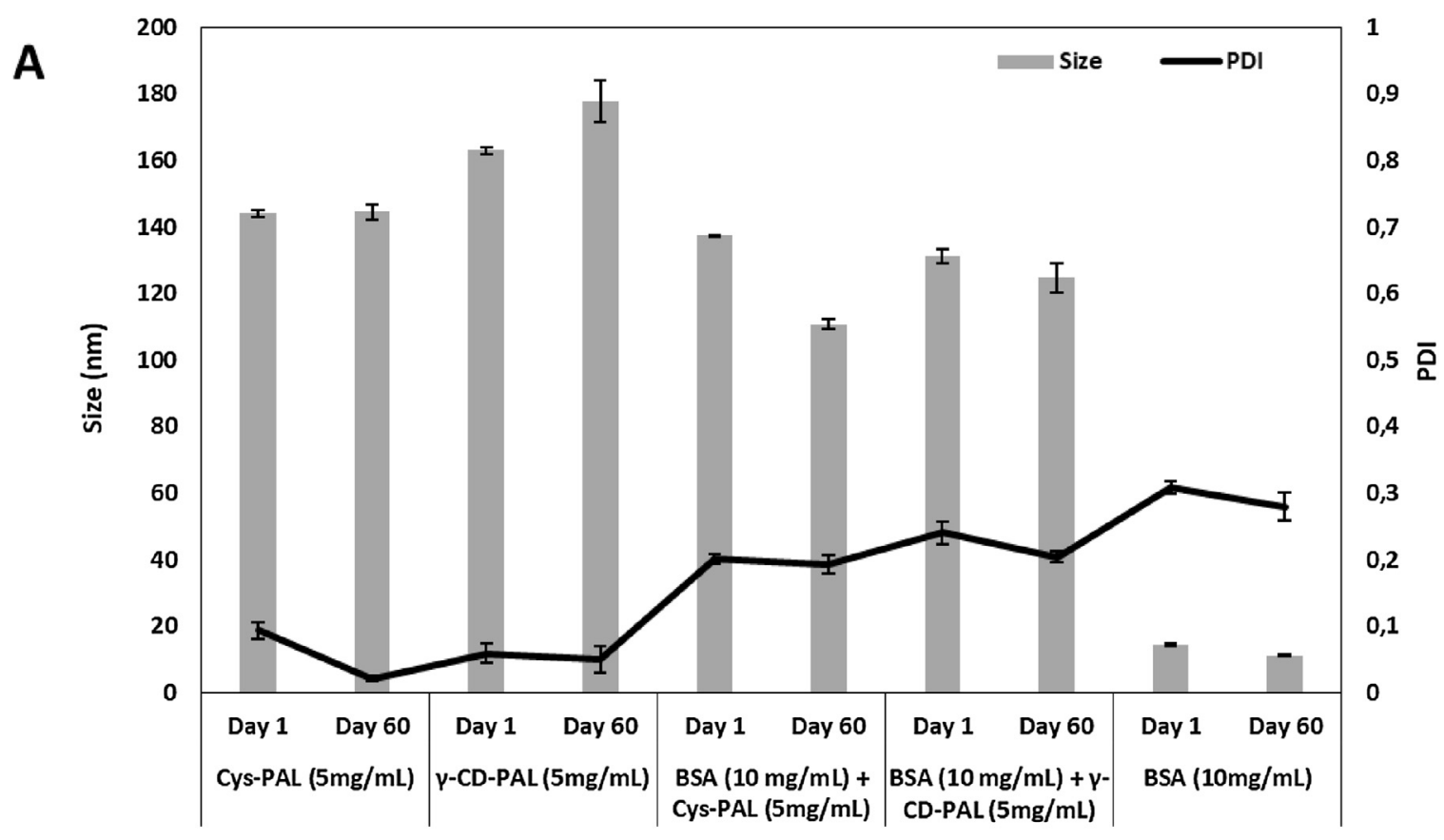

B

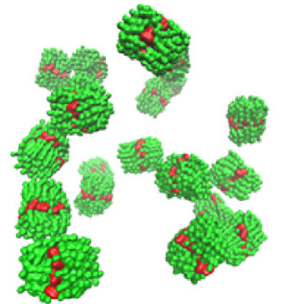

I)

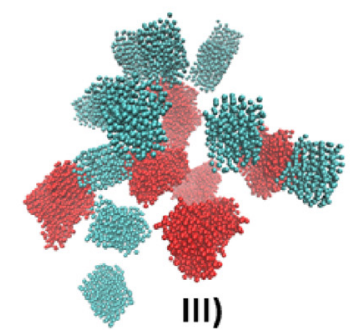

Auto aggregation of Cys-PAL units (15)

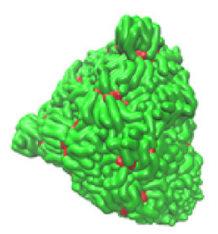

II)

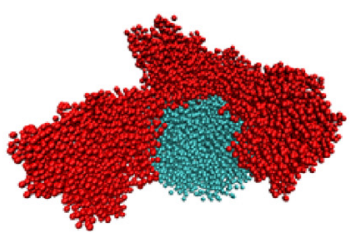

IV)

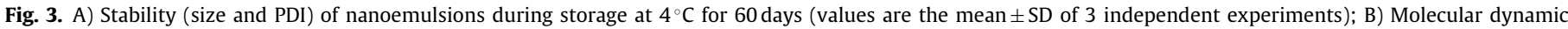

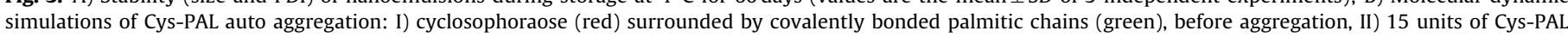

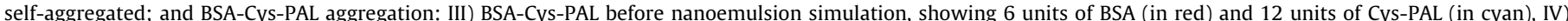

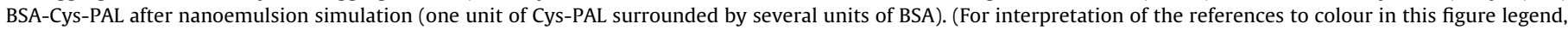
the reader is referred to the web version of this article.)

oligosaccharides with the protein. After storage for 60 days at $4{ }^{\circ} \mathrm{C}$ the surface charge of nanodroplets remains highly negative rendering high stability to the developed emulsions.

The formulations containing BSA in their composition present the lowest size values and the highest PDI. The size observed might be explained by the presence of the protein which is believed to form a shell with hydrophobic characteristics near the oilylike cyclo-oligosaccharides and hydrophilic properties near water (Fig. 3B). The PDI observed might be related with the yield of nanoemulsions formation. While samples containing only cyclooligosaccharides present nanoemulsions formation yields very close to $100 \%$, samples containing BSA might contain protein that do not contribute to the nanodroplets formation, increasing the PDI measured by DLS. These results demonstrate that the cyclooligosaccharides-PAL seems to be an effective carbohydrate-based emulsifiers. The high emulsion stability related with the low floccu- lation and/or coalescence obtained suggests many possibilities of uses as molecular devices for the development of cosmetic and/or pharmaceutic products with a long shelf life.

The size diameter of nanodroplets was also evaluated by NTA and the results were compared with the values obtained by DLS (Table 1). NTA, besides giving information about nanoemulsions concentration allowed us to eliminate the influence of small amounts of reagents that did not participate in the nanodroplets formation. The mean size diameter results obtained by both techniques follow the same tendency. Nanoemulsions containing only the modified cyclo-oligosaccharides show values of size diameter of $150-170 \mathrm{~nm}$ on both techniques. The same behaviour is observed for the samples containing BSA in their composition which demonstrate higher mean size values observed by NTA. Both techniques reveal only one peak, indicating the monodisperse character. NTA analysis allowed to quantify the nanodroplets concentration on 
Table 1

Mean size diameter ( $\mathrm{nm}$ ) and size distribution measured by DLS and NTA (values are the mean \pm SD of 3 independent experiments).

\begin{tabular}{|c|c|c|c|c|c|}
\hline \multirow[b]{2}{*}{ Nanoemulsions } & \multicolumn{2}{|l|}{ DLS } & \multicolumn{3}{|l|}{ NTA } \\
\hline & Z-average (nm) & PDI & Mean (nm) & SD & Conc. particles (particles $/ \mathrm{mL}$ ) \\
\hline Cys-PAL ( $5 \mathrm{mg} / \mathrm{mL}$ ) & $144.1 \pm 1.05$ & $0.093 \pm 0.01$ & $151.5 \pm 0.71$ & $52.5 \pm 3.5$ & $6.29 \pm 6.3(\mathrm{E} 9)$ \\
\hline$\gamma$-CD-PAL $(5 \mathrm{mg} / \mathrm{mL})$ & $162.9 \pm 1.19$ & $0.059 \pm 0.01$ & $157 \pm 14.1$ & $65 \pm 9.9$ & $8.45 \pm 2.9$ (E9) \\
\hline BSA ( $10 \mathrm{mg} / \mathrm{mL})$-Cys-PAL $(5 \mathrm{mg} / \mathrm{mL})$ & $137.4 \pm 0.23$ & $0.201 \pm 0.01$ & $173.5 \pm 3.5$ & $52 \pm 4.2$ & $11.3 \pm 0.02($ E9) \\
\hline BSA (10 mg/mL)- $\gamma$-CD-PAL ( $5 \mathrm{mg} / \mathrm{mL})$ & $131.2 \pm 1.97$ & $0.240 \pm 0.01$ & $182 \pm 7.1$ & $47.5 \pm 9.2$ & $1.91 \pm 1.1(\mathrm{E} 9)$ \\
\hline
\end{tabular}

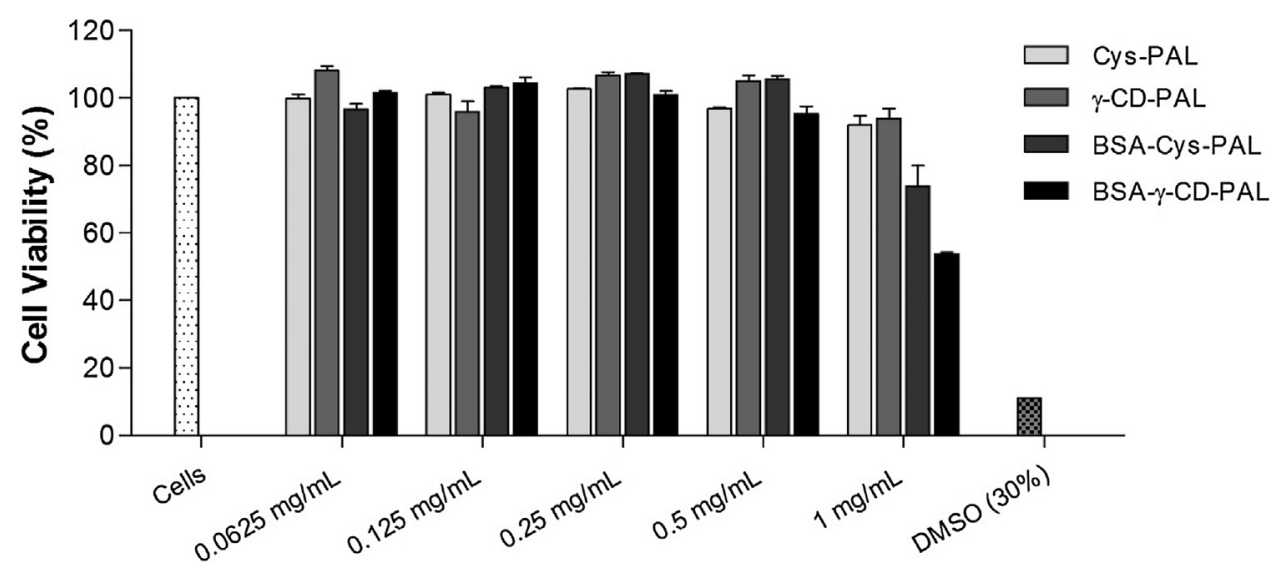

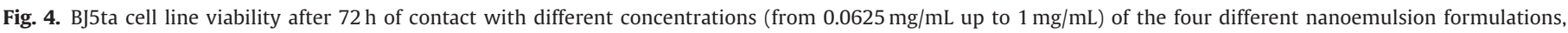

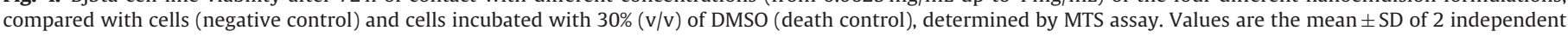
experiments.

each sample. Similar concentration of particles per $\mathrm{mL}$ were measured for both Cys and $\gamma$-CD modified cyclo-oligosaccharides. From all the samples tested, the nanoemulsions containing BSA/Cys-PAL show the highest concentration of particles after emulsification. The lowest particle concentration is observed for samples containing BSA- $\gamma$-CD-PAL.

\subsection{Cytotoxicity of nanoemulsions}

The effect of Cys-PAL ( $5 \mathrm{mg} / \mathrm{mL}), \gamma$-CD-PAL $(5 \mathrm{mg} / \mathrm{mL})$, BSA $(10 \mathrm{mg} / \mathrm{mL})-$ Cys-PAL $(5 \mathrm{mg} / \mathrm{mL})$ and BSA $(10 \mathrm{mg} / \mathrm{mL})-\gamma-C D-P A L$ ( $5 \mathrm{mg} / \mathrm{mL}$ ) on the viability human BJ5ta cells was evaluated by the MTS assay. Fig. 4 shows BJ5ta cell viability in the presence of different concentrations of nanoemulsions and DMSO at $30 \%(v / v)$ as negative control. The results demonstrated that the nanoemulsions containing the modified cyclo-oligosaccharides do not induce cytotoxicity in cells after $72 \mathrm{~h}$ of contact even at high concentration $(1 \mathrm{mg} / \mathrm{mL})$. Nanoemulsions containing BSA induced some cytotoxicity, but only for the higher concentration tested $(1 \mathrm{mg} / \mathrm{mL})$. These results indicate that the modified cyclo-oligosaccharides have potential as biocompatible encapsulating emulsifiers for further applications in pharmaceuticals, foods or cosmetics.

\subsection{Stability of nanoemulsions in the presence of esterase}

The stability of nanoemulsions in vivo is governed by several factors namely, $\mathrm{pH}$, surface charge, concentration, salts and the presence of hydrolytic enzymes [27]. Thus, we tested the stability of the developed nanoemulsions in the presence of an esterase, lipase from thermomyces lanuginosus (activity: $407 \mu \mathrm{mol} / \mathrm{ml} / \mathrm{min}$ ). The enzyme remained active during all the incubation process (esterase activity measured using the release of $p$-nitrophenol, from $p$-nitrophenyl butyrate, by absorbance at $400 \mathrm{~nm}$ ) [28]. The stability of nanoemulsions was evaluated by particle size measurements at different time points of incubation and by quantification of palmitic acid concentration after hydrolysis. This quantifica- tion was performed separating the products of hydrolysis by liquid-liquid extraction with $\mathrm{CHCl}_{3}$, followed by NMR analysis (the isolated product was firstly dissolved in DMSO- $d_{6}$ to detect the presence of palmitic acid; then, to the same sample was added $\mathrm{CDCl}_{3}$, to calculate the percentage of palmitic acid cleavage from the nanoemulsions). Table 2 presents the mean size diameter values of nanodroplets before and after enzymatic incubation for $48 \mathrm{~h}$. It is noteworthy that the size of all the samples is influenced by the same amount of catalyst in solution. It can be depicted that nanoemulsions made-up of modified cyclo-oligosaccharides are not disrupted by enzymatic action. This is confirmed by the vestigial amounts of palmitic acid ( $<1 \%$ ) detected by NMR (data not shown). Lipase catalyst was able to disrupt the nanoemulsions containing BSA as it can be observed by the results of size diameter. After incubation for $48 \mathrm{~h}$ the mean size increased as well as the PDI indicating nanoemulsions destabilization and disruption. The characteristics of these nanoemulsions make them instable per se to time storage, as it can be depicted by the higher values of PDI obtained (Fig. 3A). The self-aggregation ability of Cys-PAL give rise to nanoemulsions more robust and stable against hydrolysis (Fig. 3B).

The NMR analysis corroborate these findings. The percentages of palmitic acid cleavage are in the range of 20-55.7\% when lipase is applied (data not shown).

\subsection{Encapsulation and release of methotrexate}

As previously reported by Kim et al. [16] we also studied herein the dual properties of modified cyclo-oligosaccharides, namely encapsulation and emulsifying ability using a model compound, methotrexate (MTX). MTX is an hydrophobic-like drug in its native form acquiring hydrophilic character when solubilized at high $\mathrm{pH}$ $[29,30]$.

Taking advantage of the phase-change phenomena (from solid to oily-like liquid) we could predict an efficient encapsulation of this drug at the moment of nanodroplets formation. The encapsulation take place by mixing $10 \mathrm{mg} / \mathrm{mL}$ of MTX at the water phase 
Table 2

Comparison of particle size after emulsification $(\mathrm{t}=0 \mathrm{~h})$ and after $48 \mathrm{~h}$ in the presence of lipase (values are the mean \pm SD of 3 independent experiments).

\begin{tabular}{|c|c|c|c|c|}
\hline & \multicolumn{2}{|l|}{$\mathrm{t}_{\mathrm{oh}}$} & \multicolumn{2}{|l|}{$\mathrm{t}_{48 \mathrm{~h}}$} \\
\hline & \multicolumn{2}{|c|}{ Before incubation } & \multicolumn{2}{|l|}{ Lipase } \\
\hline & Size (nm) & PDI & Size (nm) & PDI \\
\hline Cys-PAL ( 5 mg/mL) & $146.0 \pm 1.2$ & $0.134 \pm 0.02$ & $152.2 \pm 8.7$ & $0.118 \pm 0.02$ \\
\hline BSA ( $10 \mathrm{mg} / \mathrm{mL})$-Cys-PAL ( $5 \mathrm{mg} / \mathrm{mL})$ & $106.0 \pm 0.6$ & $0.385 \pm 0.06$ & $134.4 \pm 7.1$ & $0.283 \pm 0.00$ \\
\hline$\gamma$-CD-PAL $(5 \mathrm{mg} / \mathrm{mL})$ & $172.7 \pm 2.1$ & $0.087 \pm 0.02$ & $144.5 \pm 13$ & $0.286 \pm 0.11$ \\
\hline BSA ( $10 \mathrm{mg} / \mathrm{mL})-\gamma-\mathrm{CD}-\mathrm{PAL}(5 \mathrm{mg} / \mathrm{mL})$ & $129.7 \pm 3.6$ & $0.289 \pm 0.00$ & $128.7 \pm 12$ & $0.437 \pm 0.08$ \\
\hline
\end{tabular}

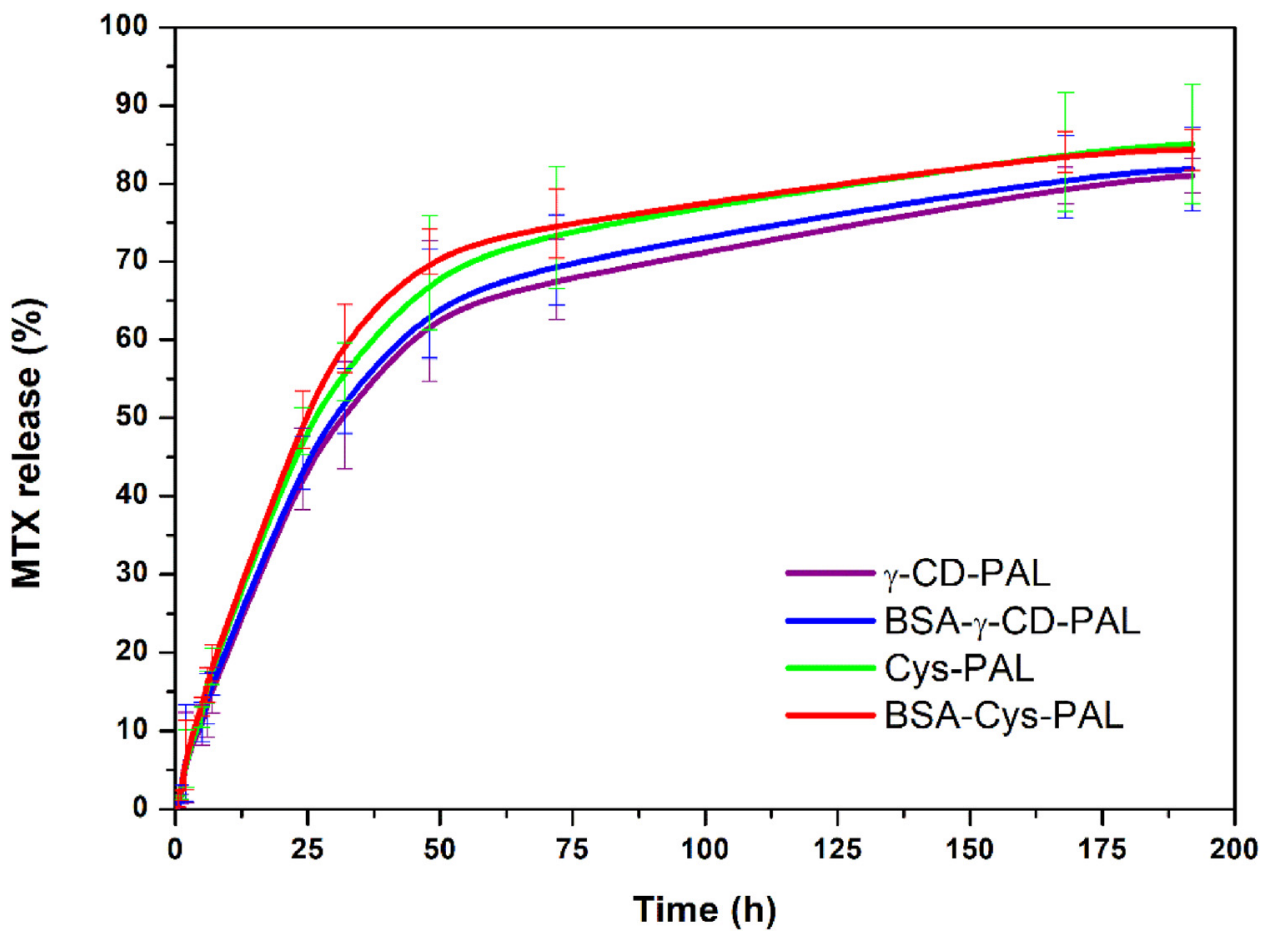

Fig. 5. Release of MTX from the nanoemulsions over time (values are the mean \pm SD of 3 independent experiments).

with the modified cyclo-oligosaccharides. The solution was heated at $60^{\circ} \mathrm{C}$ and after ultrasonication the samples showed a milky emulsion with yellow colour indicating the encapsulation and/or emulsification into the cyclo-oligosaccharides [16]. The available ester groups of the cyclo-oligosaccharides are expected to interact with the hydrophilic groups of the drug, whereas the aromatic groups would interact more easily with the hydrophobic palmitic chains introduced by synthesis with the cyclo-oligosaccharides.

By NMR spectroscopy it is possible to evaluate the molecular interactions between MTX and the cyclo-oligosaccharide nanoemulsions (Fig. S.4). The spectra of the commercial MTX evidence the aromatic peaks at $\delta_{\mathrm{H}} 8.59,7.72$ and $6.89 \mathrm{ppm}$, which undergo chemical shifts when MTX is entraped within nanoemulsions to $\delta_{\mathrm{H}} 8.54,7.66$ and $6.79 \mathrm{ppm}$. The aliphatic peaks showed up at $\delta_{\mathrm{H}} 4.79,4.32,3.18$ and $2.32-2.02 \mathrm{ppm}$ in the starting MTX, while in the nanodevices they appear at $\delta_{\mathrm{H}} 4.69,4.32,3.13$ and 2.35-2.03 ppm. Considering the small chemical shifts observed, especially of the lipophilic part of the MTX (aromatic peaks), is possible to predict that these groups preferentially interact with the hydrophobic palmitic chains of the cyclo-oligosaccharides leading to high MTX encapsulation yield. The small and narrow sized nanoemulsions containing MTX evaluated by DLS (Table S.2) corroborated this assumption. As we observed by NMR, MTX present in the solution was entrapped into nanoemulsions. We can specu- late that this entrapment is near $100 \%$ since their physicochemical properties resemble the empty nanodevices and no free MTX is detected by DLS, and thus no additional step of separation is needed.

Afterwards the release profile of the drug was traced until the amount of MTX released reached a plateau (Fig. 5). Analysing the data obtained, we can observe a burst release in the first $24 \mathrm{~h}$ of dialysis. It is also evident the release of almost $90 \%$ of the drug after 7 days of dialysis, for all the cases studied. The observations suggest that the intrinsic entrapment ability of modified cyclo-oligosaccharides functioned herein as drug encapsulators and a similar behaviour has been observed for all the cyclo-oligosaccharides tested. The encapsulation and/or entrapment ability is not, in this case, size dependent, but is related with the intrinsic phase-change properties of the cyclo-oligosaccharides and their ability to interact with amphiphilic-like compounds.

In order to evaluate the structural stability of nanoemulsions after drug release, the size and PDI of the nanodroplets was evaluated by DLS. These parameters were measured immediately after nanoemulsions formation, when occur the MTX encapsulation, and after MTX release by dialysis. The nanoemulsions containing MTX revealed to be monodisperse with small and narrow sizes (Table S.2 and Fig. S.5). After MTX release, the nanoemulsions maintained their mean size and PDI confirming their stability even after drug release. 


\section{Concluding remarks}

Herein we report for the first time the synthesis of hydrophobic cyclo-oligosaccharides by fully substitution of the hydroxyl groups with an hydrophobic compound, a palmitic chain. The emulsifier properties of these hydrophobic cyclo-oligosaccharides made possible the development of stable nanoemulsions as shown by DLS, NTA and STEM evaluations. Narrow sized, monodisperse and nontoxic nanoemulsions with great potential for amphiphilic drugs encapsulation were developed. The entrapment and delivery ability of these new hydrophobic compounds was evaluated by encapsulation of MTX. The results indicated that the intrinsic properties of modified cyclo-oligosaccharides were able to undergo emulsification in an hydrophobic environment due to their ability to acquire oily-like behaviour when heated. On the other hand, the watersoluble part of cyclo-oligosaccharides showed to be flexible and interact with the hydrophilic portion of the drug. The release of the drug revealed a sustained trend for 1 week of dialysis. Therefore, a step forward to the fabrication of nanodevices for amphiphilic drugs encapsulation and delivery for cosmetic and pharmacological purposes was accomplished.

\section{Acknowledgments}

This study was supported by the Portuguese Foundation for Science and Technology (FCT) under the scope of the strategic funding of UID/BIO/04469/2013 unit and COMPETE 2020 (POCI-01-0145-FEDER-006684) and BioTecNorte operation (NORTE-01-0145-FEDER-000004) funded by European Regional Development Fund under the scope of Norte2020 - Programa Operacional Regional do Norte. This work has received funding from the European Union Horizon 2020 research and innovation programme under grant agreement NMP-06-2015-683356 FOLSMART. Jennifer Noro also thanks to FCT- Fundação para a Ciência e a Tecnologia for funding her scholarship (SFRH/BD/121673/2016).

\section{Appendix A. Supplementary data}

Supplementary data associated with this article can be found, in the online version, at http://dx.doi.org/10.1016/j.colsurfb.2017.07. 055.

\section{References}

[1] Y. Ran, A. Jain, S.H. Yalkowsky, Solubilization and preformulation studies on PG-300995 (An anti-HIV drug), J. Pharm. Sci. 94 (2005) 297-303.

[2] T.J.M. Meerum, J.H.M. Schellens, W.W.t.B. Huinink, J.H. Beijnen, Clinical pharmacology of anticancer agents in relation to formulations and administration routes, Cancer Treat. Rev. 25 (1999) 83-101.

[3] A.F.A. Núñez, S.H. Yalkowsky, Solubilization of diazepam, J. Pharm. Sci. Technol. 52 (1998) 33-36.

[4] S.K. Han, G.Y. Kim, Y.H. Park, Solubilization of biphenyl dimethyl dicarboxylate by cosolvency, Drug Dev. Ind. Pharm. 25 (1999) 1193-1197.

[5] A.T.M. Serajuddin, Solid dispersion of poorly water-soluble drugs: early promises, subsequent problems, and recent Breakthroughs, J. Pharm. Sci. 88 (1999) 1058-1066.

[6] A. Loureiro, N.G. Azoia, A.C. Gomes, A. Cavaco-Paulo, Albumin-Based nanodevices as drug carriers, Curr. Pharm. Des. 22 (2016) 1371-1390.

[7] W.H.D. Jong, P.J.A. Borm, Drug delivery and nanoparticles: applications and hazards, Int. J. Nanomed. 3 (2008) 133-149.
[8] Z.A. Khan, R. Tripathi, B. Mishra, Methotrexate: a detailed review on drug delivery and clinical aspects, Expert Opin. Drug Deliv. 9 (2012) 151-169.

[9] S.S. Abolmaali, A. Tamaddon, G. Yousefi, K. Javidnia, R. Dinarvand, Sequential optimization of methotrexate encapsulation in micellar nano-networks of polyethyleneimine ionomer containing redox-sensitive cross-links, Int. J. Nanomed. 9 (2014) 2833-2848.

[10] P. Chakkarapani, L. Subbiah, S. Palanisamy, A. Bibiana, F. Ahrentorp, C. Jonasson, C. Johansson, Encapsulation of methotrexate loaded magnetic microcapsules for magnetic drug targeting and controlled drug release, J. Magn. Magn. Mater 380 (2015) 285-294.

[11] S. Massadeh, M. Alaamery, S. Al-Qatanani, S. Alarifi, S. Bawazeer, Y. Alyafee, Synthesis of protein-coated biocompatible methotrexate-loaded PLA-PEG-PLA nanoparticles for breast cancer treatment, Nano Rev. Exp. 7 (2017) 31996-32005.

[12] D. Soto-Castro, J.A. Cruz-Morales, M.T. Ramirez Apan, P. Guadarrama, Solubilization and anticancer-activity enhancement of Methotrexate by novel dendrimeric nanodevices synthesized in one-step reaction, Bioorg. Chem. 41-42 (2012) 13-21.

[13] A. Loureiro, E. Nogueira, N.G. Azoia, M.P. Sarria, A.S. Abreu, U. Shimanovich, A. Rollett, J. Harmark, H. Hebert, G. Guebitz, G.J. Bernardes, A. Preto, A.C. Gomes, A. Cavaco-Paulo, Size controlled protein nanoemulsions for active targeting of folate receptor positive cells, Colloids Surf. B: Biointerfaces 135 (2015) 90-98.

[14] A. Loureiro, A.S. Abreu, M.P. Sarria, M.C.O. Figueiredo, L.M. Saraiva, G.J.L. Bernardes, A.C. Gomes, A. Cavaco-Paulo, Functionalized protein nanoemulsions by incorporation of chemically modified BSA, RSC Adv. 5 (2015) 4976-4983.

[15] B. Gidwani, A. Vyas, A comprehensive review on cyclodextrin-Based carriers for delivery of chemotherapeutic cytotoxic anticancer drugs, BioMed Res. Int. 2015 (2015) 1-15

[16] H. Kim, S.D. Dindulkar, D. Jeong, S. Park, B.-H. Jun, E. Cho, S. Jung, A synthetic encapsulating emulsifier using complex-forming pentacosadiynoyl cyclosophoraoses (cyclic B-(1, 2)-D-glucan), J. Ind. Eng. Chem. 44 (2016) 195-203.

[17] Y.-E. Kwon, E.-A. Cho, I.-S. Lee, S.-H. Jung, Synthesis and characterization of butyryl cyclosophoraose, and its inclusion complexation behavior for some flavonoids, Bull. Korean Chem. Soc. 32 (2011) 2779-2782.

[18] K. Koizumi, Y. Okada, S. Horiyama, T. Utamura, T. Higashiura, M. Ikeda, Preparation of Cyclosophoraose-A and its complex-forming ability, J. Incl. Phenom. 2 (1984) 891-899.

[19] S.D. Dindulkar, D. Jeong, E. Cho, D. Kim, S. Jung, Microbial cyclosophoraose as a catalyst for the synthesis of diversified indolyl $4 \mathrm{H}$-chromenes via one-pot three component reactions in water, Green Chem. 18 (2016) 3620-3627.

[20] H. Kim, J.M. Choi, Y. Choi, M.N. Tahir, Y.-H. Yang, E. Cho, S. Jung, Enhanced solubility of galangin based on the complexation with methylated microbial cyclosophoraoses, J. Incl. Phenom. Macrocycl. Chem. 79 (2014) 291-300.

[21] R. Silva, H. Ferreira, N.G. Azoia, U. Shimanovich, G. Freddi, A. Gedanken, A. Cavaco-Paulo, Insights on the mechanism of formation of protein microspheres in a biphasic system, Mol. Pharm. 9 (2012) 3079-3088.

[22] M.W. Breedveld, L.P.T.M. Zevenhuizen, A.J.B. Zehnder, Excessive excretion of cyclic 13-(1,2)-glucan by rhizobium trifolii TA-1, Appl. Environ. Microbiol. 56 (1990) 2080-2086

[23] C. Kwon, Y. Choi, D. Jeong, J.G. Kim, J.M. Choi, S. Chun, S. Park, S. Jung, Inclusion complexation of naproxen with cyclosophoraoses and succinylated cyclosophoraoses in different pH environments, J. Incl. Phenom. Macrocycl. Chem. 74 (2012) 325-333.

[24] S. Pronk, S. Páll, R. Schulz, P. Larsson, P. Bjelkmar, R. Apostolov, M.R. Shirts, J.C. Smith, P.M. Kasson, D. van der Spoel, B. Hess, E. Lindahl, GROMACS 4.5: high-throughput and highly parallel open source molecular simulation toolkit, Bioinformatics 29 (2013) 845-854.

[25] B. Hess, H. Bekker, H.J.C. Berendsen, J.G.E.M. Fraaije, LINCS: A linear constraint solver for molecular simulations, J. Comput. Chem. 18 (1997) 1463-1472.

[26] H.J.C. Berendsen, J.P.M. Postma, W.F.V. Gunsteren, A. DiNola, J.R. Haak, Molecular dynamics with coupling to an external bath, J. Chem. Phys. 81 (1984) 3684-3690

[27] Z. Wu, B. Zhang, B. Yan, Regulation of enzyme activity through interactions with nanoparticles, Int. J. Mol. Sci. 10 (2009) 4198-4209.

[28] T. Matamá, F. Vaz, G.M. Gübitz, A. Cavaco-Paulo, The effect of additives and mechanical agitation in surface modification of acrylic fibres by cutinase and esterase, Biotechnol. J. 1 (2006) 842-849.

[29] S.K. Ramamoorthy, R. Hephziba, Acute renal failure post high dose methotrexate infusion successfully managed with high dose folinic acid and high flux dialysis, Indian J. Hematol. Blood Transfus. 29 (2013) 90-92.

[30] O.M. Al-Quteimat, M.A. Al-Badaineh, Practical issues with high dose methotrexate therapy, Saudi Pharm. J. 22 (2014) 385-387. 